\title{
Research on the Translation of Sichuan Wine Advertisement under the Perspective of Memetics
}

\author{
Li Xiao \\ School of Foreign Languages, Southwest Medical University, Lu Zhou Si Chuan, China \\ E-mail: xiaoli60711@163.com
}

Keywords: Transliteration, Sichuan Wine Advertisement, Memetics

\begin{abstract}
In cultural evolution, meme is a replication factor similar to that played by genes in biological evolution, and is a unit of cultural transmission. Any language unit that can be propagated by imitation is a linguistic meme. Memes has the strongest stability, the highest usage rate, and the longest survival time. On the basis of memetics theory, the foreign propaganda language of Sichuan wine is the main carrier of the cultural transmission of Sichuan wine. It studies the languages used by some famous Sichuan wine companies in the spirit of enterprise, cultural ideas and television advertisements, in order to mold a stable strong language Memes and provide reference to promote the spread of Sichuan wine culture.
\end{abstract}

\section{Introduction}

Memetics, as a unit of cultural transmission, survive by copying and spreading. Language is one of the carriers of memes. Language itself is also a meme. In terms of language, phonetics, semantics, grammar, words, etc. are messages that are passed through imitation and can naturally be seen as memes. Any part of the language, as long as copied and disseminated through imitation, may become a language meme. The successful meme, like successful replicons has the characteristics of copying-fidelity, fecundity, and longevity. Fidelity is the essence of memes that often retain their original meme during the copying process. Productivity refers to the spread of memes and the spread of a wide range of speed. Longevity refers to the memetic long survival time, that is, in the paper or people's minds spread for a long time. According to the above three characteristics, memes can be divided into strong memes and memes. A strong meme copy of high fidelity, the chance of being copied, spread a wide range of long survival time. The weak memes are the opposite. Language memes can also be divided into strong language memes and weak language memes. According to the degree of strength, strong language memes are divided into stable strong language memes and changes in strong language memes. Stable strong language Memes the strongest stability, the highest utilization rate, the longest survival time. The strong language memes of change are high fidelity and high frequency of copying in a certain period, but they are quiet or even disappear after a certain period of time, and the active time is shorter than that of strong and stable language.

Among all means of communication, language is the most effective means of memetic transmission. Effective language use is for better memetic transmission. Memetics should be successfully disseminated. The first is to conform to and conform to the nature of human beings as imitators and selectors. It can make people have a unique emotional experience; the second is to have its own characteristics of easy to disseminate, easy to imitate and memory, can enter the host "self" memes can easily be successfully spread; the third is to enter the appropriate host. As a mememe, linguistic memes also survive through reproduction and dissemination, and likewise support the system of rules of evolution based on mutation, selection, and maintenance (or inheritance). The continuous process of variation, selection, and maintenance of linguistic memes drives the evolution of languages. Through the selection of variant of linguistic memes, some of the requirements for the successful propagation of memes can be satisfied, and correspondingly, the success rate of memetic transmission can be improved. 


\section{Communication Ways and Pragmatic Effect}

The memes, which convey the content of the information, are stored in people's brains and can be compared to the memes of genotypes. The mesogenic modes of transmission include the same information being transmitted in the same form as the original message and the same message. The propagation of linguistic memes is mainly manifested as the same message being transmitted in the shape of an alien. Proper selection of variants produced by deformation of modulo can enhance the novelty of language expression and increase the attention of information and the success rate of communication. Memetic phenotypic mode of transmission is memetic because of the formation of complex memes spread, usually weak memes with a strong memetic influence to improve their viability. Phenotypic transmission of linguistic memes is the propagation of linguistic memes in a combined variant. Merger and communication is to convey new information through familiar words, sentences, words and texts that express specific information. Appropriate use of merger and communication, both enhance the novelty of language expression, but also give people a sense of familiarity and intimacy.

Interpretation of language memes need context. Situational contexts, linguistic contexts, one or more contexts within a cognitive context may induce or stimulate the replication and dissemination of the language memes. Situational contexts include contextual time, space and place, historical and realistic, and even virtual people and events, as well as all the status quo at the time of an event or discourse. Linguistic context refers to the context that understands the meaning of a word and common or cultural knowledge that is mutually understood. Cognitive context is the outcome of processing and processing of contextual situations by people and is the accumulated experience of life. Different cultural environments, different geographical environments, different nationalities, different religions and different social living environments have a great influence on the diffusion of language memes. Language memes are the product of social practice and are often closely linked with specific social events. New social practice activities will promote the emergence of new memes. [6] From a philosophical point of view, the word itself is not only action, but also may lead to new actions, therefore, language memes are the potential motivation of social practice.

\section{Sichuan Foreign Propaganda Language}

Memetics as a unit of cultural transmission is by copying, spreading and survival. As mentioned above, language is the most effective means of memetic transmission among all means of communication. Outward propaganda language occupies a decisive position in the various means of propaganda of Sichuan wine enterprises. The propaganda language of Sichuan alcohol is the main carrier of the cultural transmission of Sichuan wine, and the effective use of language is to better disseminate memetics. The following will be from the perspective of memetics theory analysis of some famous Sichuan wine business language.

Entrepreneurial spirit refers to the common inner attitude, ideological realm and ideal pursuit that employees have, which expresses the spirit of the enterprise and the style of the enterprise. Wuliangye's entrepreneurial spirit is "pioneering and innovative, competitive, hard work, progressive." Jiannanchun's entrepreneurial spirit is "unity, high quality, pioneering and innovative, dedicated, dedication." Luzhou Laojiao's entrepreneurial spirit is "respect and dedication, innovation and excellence." Tuopai's entrepreneurial spirit is "seize the day." Langjiu spirit of enterprise is "higher, faster, stronger Chinese Lang spirit." From the perspective of memetics, entrepreneurial language should become a powerful language meme in language memes, which should be stable, highly utilized and have a long time to survive. In contemporary China, "innovation" frequently appears. As the basic vocabulary in language, "innovation" is frequently used and accurately reproduced, which is a powerful language memes. Therefore, Wuliangye, Jiannanchun and Luzhou use the term "innovation" Is conducive to the spread of entrepreneurial spirit. Languages from classical discourse or famous articles are well-known due to their high frequency of copying, and they are also stable strong language memes. Mao Zedong's "Comrade Jiang Hong and Comrade Guo Moruo" in a classic sentence is "a thousand years too long, seize the 
day and night", Tuopai "contests for the day" comes from this classic, is naturally a strong meme form of stability. Language in the idioms, aphorisms, aphorisms, such as the use of high frequency is with a more productive and longevity. "Higher, faster and stronger" is the famous motto of the Olympic Movement. Langjiu used this expression in the spirit of enterprise and naturally became a powerful language meme. Strong language memes need to be highly simplified, with the most simple form of rich content or concept, there must be a core idea or concept. From this perspective, the wording of Wuliangye and Jiannanchun is slightly cumbersome. In order to make the spirit of the enterprise easy to be memorized by the modelers (people), in order to make it easier for the modelers (people) to understand the core concepts of corporate communication, think about using cleaner, more communication-friendly languages.

Corporate culture is the concept of business formed by its own characteristics of the business purposes, values and ethical conduct of a comprehensive, is the inherent quality of enterprises. Wuliangye's cultural philosophy is "to create value for customers, to achieve the ideal for employees to jointly create a better tomorrow for the company," Jiannanchun's cultural concept is "to contribute to the country, creating benefits for the enterprise, to create a better life for themselves," Luzhou cultural concept of the cellar is "to live a happy and full life in the splendid culture of wine in China." Tuopai's cultural concept is "people-oriented, scientific development, safety and harmony, truth-seeking quality, seeking perfection for people and seeking beauty through life" , Langjiu culture concept language is "three gas (righteousness, atmosphere, domineering) two sense (mystery, fashion sense)." From the perspective of memetics, the terms "value, ideal, beauty, tomorrow, country, enterprise, own, culture, human being, happiness, life, people, development, safety and quality" are all the basic words in our daily life. "People-oriented and scientific development" belongs to the idiom used in Chinese society today. With its prolific and prolific nature, with the loyalty of reproduction, it naturally becomes a powerful language meme. Therefore, Wuliangye, Jiannanchun, Luzhou Laojiao and Tuopai culture concept terms strong stability, high utilization rate, with strong language memes characteristics. The concept of Langjiu's culture is simple and concise. However, it does not directly understand the connotation expressed by the language and thus affects the reproduction and dissemination of the language. To make language easier to understand and spread, companies can consider using a clearer language to convey their cultural beliefs.

Example: "Tang Dynasty Court Wine, Sheng Jian Jian Nan Chun," succinct language, but also passed on to inherit and promote the Chinese traditional culture, so that die host (people) are now living in the prosperity of the great rejuvenation of the Chinese nation's fortune, belong Stable strong language memes. "Chinese liquor value model" uses the basic vocabulary of the language, copy and use of high frequency, search Baidu website, the use of "value model" expressed many, for example, electric vehicle Hydi's "industry benchmark, a model of value," Camry The "peak technology, a model of reproduction value", glorious Oriental Plaza "Chang An Avenue value model" and so on. Visible, Jiannanchun ad language is a strong language memes. "The power of feeling the years with taste" is the spread of memetics. In general, people use "heart" to feel it. However, the term changes "heart" to "taste" and enhance the uniqueness of language. There are Help to improve the advertising attention and the success rate of dissemination. "Millennium respect one's career, a thousand brews a spring" is a combined transmission of language memes, "dedication" itself is the basic vocabulary in the language, China has always been the "professional music group" of the traditional virtues through the use of vocabulary deviation and configuration Deviation from the form, to "respect one industry", which is a manifestation of the strong language memes of prolific. "Dedication" is also one of the entrepreneurial languages of Jiannanchun, therefore, this advertising phrase combined with entrepreneurial spirit is a successful language meme.

\section{Conclusion}

With the acceleration of the process of global economic integration, increasing competition in the market and the era of "wine is not afraid of deep alley" no longer exists. Outreach work is more 
and more important in the development of Sichuan wine enterprises. Outward propaganda means, propaganda language occupies a pivotal position, is the main carrier of Sichuan wine culture. Based on the meso-based perspective, effective linguistic use is better for memetic transmission. According to the standards of fidelity, prolificity and longevity, Sichuan wine enterprises should pay attention to the choice of propaganda language and strive to create a stable strong language meme.

\section{Acknowledgements}

Fund Project: Sichuan Provincial Department of Education base issue - Sichuan International Wine Cultural Communication Center, Project Number: CJCB2017-13

\section{References}

[1] Xu Juan. Pragmatic analysis of the buzzword "I think quiet" in the perspective of memetics [J]. Modern Language (Language Studies) .2017 (09)

[2] Liu Yanhua, Cao Jing. Journal of Yanbian Education College Journal of Yanbian Education College Journal of Yanbian University (Philosophy and Social Sciences Edition)

[3] Huang You. Language in the "non-act" phenomenon of the meme of interpretation [J]. Journal of Taishan University. 2017 (05)

[4] Cao Shuping. The interpretation of the model name in the translation of brand name [J]. Vocational Education and Economic Research. 2011 (04)

[5] Hu Guang, Ai Yuting.Study on Conflict Discourse from the Perspective of Phenomenology [J]. Foreign Language Art Education Research 2009 (04)

[6] Liu Pinglei. Implications of memetics on foreign language translation teaching [J]. Journal of Jiangxi Blue Sky College 2008 (01) 